\title{
SÍNDROME DEL TÚNEL DEL CARPO: UNA ENFERMEDAD SOCIAL MODERNA QUE REQUIERE INTERVENCIÓN OCUPACIONAL
}

* Claudia Andrea Urbina

\section{RESUMEN}

El Síndrome del Túnel del Carpo es el trauma acumulativo más frecuente y la patología que los usuarios de computador sufren con mayor regularidad. En Terapia Ocupacional se constituye un gran porcentaje de los motivos de consulta. La intervención ocupacional se inicia con una valoración a través de la observación de diferentes signos, la recreación de los sintomas por medio de pruebas sensitivas y motrices y la confirmación del diagnostico con la utilización de pruebas ocupacionales, así como la valoración de la influencia sobre la funcionalidad e independencia en la ejecución de las diferentes actividades de la vida diaria. El tratamiento tiene como objetivo minimizar los efectos de la inflamación, reducir el dolor y mantener el deslizamiento normal de los tendones flexores dentro del túnel carpiano mediante un programa de ferulaje, modificación de la actividad, técnicas de protección articular y de conservación de energía y relajación.

Palabras clave: Síndrome del Túnel del Carpo (SCT), protección articular, conservación de energía, modificación de la actividad, férulas.

\section{INTRODUCCIÓN}

Q1 Síndrome del Túnel del Carpo es el trastorno de trauma acumulativo más frecuente. CLa mitad de los casos del Síndrome del Túnel del Carpo (STC) se producen por movimientos repetitivos de flexo extensión de muñeca, en la mayoría de los casos por causa del trabajo, en especial las labores domésticas, donde es necesario realizar actividades como cargar objetos, lavar, escurrir o torcer el trapero, amasar y realizar labores manuales 
como bordar y tejer, como se demostró en el Estudio Publicado en la Revista de la Ocupación Humana, escrito por Santaella y colaboradoras, en 1998, realizando una comparación entre de dos férulas de distinto material en cuanto a efectividad en amas de casa con Síndrome del Túnel Carpiano

En el 2001, el Síndrome del Túnel del Carpo (STC) fue reportado por la Empresa Lokhheed Martín, en dos terceras partes de las enfermedades laborales en Norte América; es además el trauma acumulativo que más sufren los usuarios de los computadores, como se muestra en la Revista Enter de febrero de 2003, lo que hace que el Síndrome del Tunel del Carpo (STC), sea la enfermedad profesional de la época.

En la consulta del Servicio de Terapia Ocupacional del Hospital Militar Central de los años 2000, 2001 y 2002, se encontró que dicha patología se presentó en una proporción de mujeres a hombres de 8:1; de la mujeres, se determinó que un $45 \%$ eran amas de casa, el $15 \%$ secretarias, el 10\% modistas, el $6 \%$ laboraban como esteticistas, enfermeras y cocineras, y el restante $12 \%$ repartido en otros oficios como el de estudiantes y profesoras.

Las mujeres con mayor incidencia fueron aquellas entre los 51 y 60 años de edad, seguidas por las mujeres entre los 41 y 50 años. En todos los casos se utilizaron palmetas cortas para el Síndrome del Túnel del Carpo, elaboradas en promedio cada una de ellas en tres sesiones de 30 minutos, es decir 90 minutos. El costo del material y el gasto anual se muestra en el Cuadro No. 1, según la estadística del Hospital Militar Central del Servicio de Terapia Ocupacional.

\begin{tabular}{lccc} 
AÑ̃O & No. PACIENTES & No. FERULAS & \multicolumn{1}{c}{ COSTO } \\
2000 & 92 & 266 & $\$ 16,824,500$ \\
\hline 2001 & 112 & 177 & $\$ 11,195,250$ \\
\hline 2002 & 79 & 108 & $\$ 6,831,000$ \\
\hline TOTAL & $\mathbf{2 8 3}$ & $\mathbf{5 5 1}$ & $\$ \mathbf{3 4 , 8 5 0 , 7 5 0}$ \\
\hline
\end{tabular}

\section{Cuadro $N^{\mathrm{a}} 1$}

El tratamiento tradicional conservador para los usuarios con STC, implica la utilización de ferulaje, donde se inmoviliza la muñeca para evitar la flexo extensión de la misma y producir la disminución del edema a este nivel, y por consecuencia la descompresión del nervio mediano, y la disminución de los síntomas.

Los terapeutas ocupacionales son quienes, además de implementar un tratamiento de enfoque biomecánico, en donde se incluyen las técnicas de protección articular, ahorro de energía, relajación, así como de acondicionamiento de esta zona del miembro superior, se encargan de diseñar, elaborar y aplicar las férulas y adaptaciones que se requieren para cada caso en particular.

Para el Síndrome del Túnel del Carpo se están diseñando y aplicando férulas en material termoplástico, de uso diurno y nocturno por tres semanas a partir de la primera visita al servicio de Terapia Ocupacional, seguido por uso diurno exclusivamente, las siguientes tres semanas. En este proceso se ha evidenciado disminución de los síntomas, expresados por cada uno de los usuarios al finalizar las tres primeras semanas cuando el usuario ha sido instruido en cuanto a las técnicas de Protección Articular, Ahorro de energía, de Relajación y de acondicionamiento de la musculatura de los miembros superiores. Los resultados en cuanto a la disminución de la sintomatología aunque fueron reportados por cada uno de los pacientes y evidenciados por la reanudación de sus actividades laborales, no fueron documentados en su momento por lo que no son medibles en la actualidad.

\section{Patologia del síndrome de TÚNEL DEL CARPO}

El STC es una patología que afecta la mano, provocada por el aumento de presión en el túnel del carpo que produce la compresión del nervio mediano.

En Colombia, según lo muestra la Revista Tribuna Medica, en su edicion de Mayo de 1996, es considerado un problema clínico común en especial después de la quinta década de la vida siendo la incidencia en mujeres del 3\% y en hombres del 2\%. En la población general alrededor del $15 \%$ de las personas manifiestan sintomatología compatible con STC. 
El nervio mediano y sus ramas motoras inervan los músculos del antebrazo que permiten la flexión de las articulaciones interfalangicas proximales de los dedos, flexión del pulgar y de la muñeca, así como la pronación del antebrazo.

A nivel de la muñeca, este nervio pasa a través del túnel carpiano y en su salida, la rama motora pasa a los músculos de la región tenar que proporcionan los movimientos de abducción, flexión y oposición del pulgar. Termina con los nervios digitales en los dedos índice y medio, que también inervan los lumbricales primero y segundo de estos dedos respectivamente, dando la posibilidad de realizar la flexión de las articulaciones metacarpofalángicas y extensión de las interfalángicas.

El nervio mediano al salir del túnel del carpo da también inervación sensitiva a la zona radial y central de la palma de la mano y a la cara palmar de los dedos uno, dos, tres y mitad radial del cuarto así como en la cara dorsal zona radial distal del segundo, tercero y mitad radial del cuarto. La zona autónoma del nervio mediano en la mano es la falange distal del segundo dedo en la cara palmar.

El túnel carpiano es una estructura anatómica localizada en la cara anterior de la muñeca. Como su nombre indica, es una especie de corredor atravesado por elementos importantes para la movilización y sensibilidad de la mano. El túnel está limitado en la parte posterior de cubital a radial por los huesos del carpo: el pisiforme, piramidal, semilunar y escafoides; el nervio mediano, pasa por el centro de este corredor. En la parte anterior esta limitado por el ligamento transverso del carpo que contiene además los tendones del flexor digitorum profundus y flexor digitorum sublimis; el tendón del flexor carpis radialis y flexor pollicis longus. (Revisión del volumen de Noviembre de 1992, de la Revista Hand Clinics).

\section{Etiología}

La causa más frecuente es la tenosinovitis estenosante de los tendones flexores de los dedos, por lo general bilateral, que impide su deslizamiento a través del túnel (Revista ILADIBA,Septiembre de
2002). La ocupación laboral tiene estrecha relación con la aparición del STC, en especial, la ejecución de acciones manuales repetitivas, en nuestro medio específicamente ocupaciones como las de procesamiento de comidas, manufacturas, trabajo de construcción, costura y trabajo en computador, como es evidente en el artículo sobre la fisiopatología del STC, de la Hand Clinics de Mayo de 1996, o en deportes en los que además de movimientos repetitivos, se requiere la aplicación de cierto grado de fuerza, tales como el Squash y el Golf.

Los movimientos que contribuyen a la Tenosinovitis estenosante son la flexión y la extensión de la muñeca, éstos elevan la presión tisular de los líquidos en el túnel carpiano. Los movimientos repetítivos que incluyan flexión, extensión y desviación cubital de la muñeca, particularmente cuando se combinan con la acción de agarrar alguna herramienta, pueden causar los síntomas del Síndrome del Túnel del Carpo. Esas afecciones traumáticas acumulativas afectan a menudo a personas en ocupaciones específicas como empacadores de carne, cajeras, costureras, músicos o trabajadores en línea de ensamblaje, especialmente cuando estas tareas repetitivas también incluyen exposición a vibraciones de la muñeca o de la mano. Las personas que utilizan sillas de ruedas también se ven afectadas con esta patología. Casi la mitad de los parapléjicos tienen los signos y la sintomatología debido a que la mayoría de las actividades cotidianas que les exigen levantarse desde la posición sedente, se llevan a cabo con las muñecas en extensión máxima (Push ups), lo que aumenta la presión de los tejidos dentro del túnel carpiano.

\section{El Síndrome del Túnel del Carpo y la ERgonomía}

Tunner en 1994, estableció que el STC está influenciado por la nueva tecnología y por el aumento de trabajo físico, movimiento y esfuerzo concentrado en la muñeca. Por ejemplo, la carga estática está vista como un factor significativo debido al mal diseño de 
los equipos y herramientas, así mismo los descansos inadecuados y a destiempo, la tensión permanente en los grupos musculares extrínsecos de la mano y los disturbios emocionales de los trabajadores modernos, impiden la recuperación de la fatiga de esta zona osteomuscular.( Rol del Terapeuta Ocupacional, de la Revista British Journal of Occupational Therapy, de Febrero de 1997).

Ciertos tipos de posturas incrementan el riesgo del STC, incluyendo manos en el teclado, carencia de soporte de brazos, y las tareas de organización.

\section{Influencia Psicológica del SínDROME DEL TÚNEL DEL CARPO}

Los psicólogos están seguros que a pesar de que el STC es una afección física, los factores psicológicos contribuyen a su desarrollo y mantenimiento. El daño es parte de la expresión de un gran problema social. Así, Mulledy y Bryy (1990) demostraron que la tensión y el estrés son factores importantes, ya que algunas de las personas que padecen esta patología son incapaces de manejarlos adecuadamente, teniendo incremento en la sintomatología general. Spillane y Devesa (1997) demostraron que lo anterior trae repercusiones en el desempeño laboral de estas personas. Kiesler y Find (1998) describieron cómo el desarrollo de la nueva tecnología, tiende a incrementar presiones de trabajo, al igual que la subespecialización, los cambios de condiciones laborales, la rapidez y fuerza en el movimiento de las manos, incrementando las presiones psicológicas del usuario, con una incapacidad marcada en la corrección de las situaciones antes anotadas, obteniéndose como resultado el incremento de los trabajadores que padecen y se incapacitan por el Síndrome del Túnel del Carpo por tiempo prolongado. Otras causas son amiloidosis, secuelas anatómicas de intervenciones médicas o quirúrgicas, lesiones de discos cervicales. espondilosis cervical, trastornos anatómicos congénitos, micro traumatismos de repetición, neuropatía periférica, posturas mantenidas, esguinces repetidos, tendinitis - tenosinovitis, traumatismos de la muñeca (fractura de Colles) y variantes anatómicas: músculos anómalos o lumbricales hipertrofiados.

Los síntomas varían en los casos agudos y crónicos y en ocasiones pueden no aparecer, los que se encuentran con mayor frecuencia son:

- Entumecimiento, sensación urente y hormigueo de los tres primeros dedos

- Parestesias nocturnas

- Disestesias o hiperestesias provocadas por el frío

- Los dedos pulgar, índice, medio y la mitad radial del anular, todos por su cara palmar, sufren disestesias que se presentan con mayor frecuencia en la noche.

- Síntomas nocturnos que mejoran con la actividad suave y empeoran con la fuerte.

- Irradiaciones a nivel proximal (codo y hombro) sobre la zona por donde transcurre el nervio Mediano.

- Anestesia de los dedos pulgar, índice, medio y mitad radial del anular.

- Entorpecimiento de la sensibilidad de los dedos índice, medio y anular

- Pérdida de destreza motriz fina o torpeza manual

- En una etapa tardía hay atrofia de la región tenar y pérdida de la coordinación para oponer el pulgar.

- Reproducción de los síntomas por flexión o extensión duraderas de la muñeca.

- Alivio del dolor al inmovilizar a muñeca en posición neutra.

- Prolongación de la velocidad de conducción nerviosa durante estudios de Electromiografía.

\section{Diagnóstico del Síndrome del Túnel del Carpo}

Éste se hace con la recolección de los síntomas y además se deben indagar los antecedentes de la persona, para establecer un posible riesgo o una predisposición ante esta patología

Se pueden aplicar los siguientes exámenes físicos (Tribuna Médica, Mayo de 1996). 


\section{ARTÍCULOS INÉDITOS}

\section{SignO dE TINNEL}

La prueba de percusión del nervio o prueba de Tinnel se lleva a cabo percutiendo ligeramente la piel a lo largo del trayecto del nervio mediano, comenzando próximamente y moviéndose distalmente. Al llegar al punto donde hay compresión del nervio, el paciente experimenta una sensación de cosquilleo. Se encontró esta prueba positiva en $70 \%$ de los pacientes en que se diagnosticó STC

\section{Signo de Phalen}

Prueba de flexión de Phalen se lleva a cabo colocando el codo del paciente en una mesa con el antebrazo en posición vertical y permitiendo que la mano caiga por gravedad doblando la muñeca, en extensión extrema La sensación de adormecimiento o cosquilleo en las áreas inervadas por el mediano dentro de los 60 segundos siguientes constituye prueba positiva. Aproximadamente $70 \%$ de los pacientes con STC presentan esta prueba positiva.

También se pueden llevar a cabo pruebas paraclínicas como el electromiograma, la radiografía y la tomografía axial computarizada.

\section{Pruebas de Sensibilidad}

\section{Pruebas de densidad de la inervación}

Estas pruebas miden la función de múltiples áreas superpuestas de receptores sensoriales periféricos y dependen en gran parte de la integración cortical. Permanecen normales aun cuando exista disfunción nerviosa moderadamente avanzada. La pérdida de discriminación entre dos puntos dentro del dermatoma del nervio mediano, así como atrofia de la región tenar, ocurren en una etapa tardía; estos son signos altamente específicos pero se desarrollan tiempo después del inicio de la lesión.

La prueba estática de discriminación de dos puntos de Weber, o la mejor conocida de estas pruebas de densidad de inervación, y en esta prueba las cifras mayores de $3 \mathrm{~mm}$ son consideradas anormales para la mano.
Las pruebas de umbral sirven para evaluar fibras nerviosas únicas que inervan una o varias células receptoras; son pruebas de densidad de inervación para detectar disfunción nerviosa periférica temprana.

La prueba más conocida de este estilo es la aplicación del test de monofilamentos de SemmesWeinstein, donde se hace rebotar tres veces varios filamentos de diferente tamaños, de manera que éstos queden perpendiculares a la superficie palmar del dedo índice, con presión suficiente como para que se arquee algo el filamento. Cuando el paciente, con los ojos cerrados, no pueda identificar que ha sido tocado con un filamento de cifras de 2.83 unidades (una unidad $=\log 10[\mathrm{mg}]$ de la fuerza requerida para arquear un filamento) o mayores, se considera una lesión obstructiva en la zona sensitiva del nervio mediano. Un estudio comparativo de las pruebas de sensibilidad en pacientes con STC halló la prueba de monofilamentos de Semmes-Weinstein anormal en $83 \%$ de los mismos; en cambio la prueba de discriminación de dos puntos fue anormal únicamente en un $22 \%$ de los que padecían el STC.

\section{Pruebas motrices}

Resulta fácil valorar la fuerza de los dos músculos afectados por el STC: el abductor pollicis brevis y el opponnens pollicis. El paciente coloca el dorso de la mano en una mesa y dirige el pulgar hacia el techo. En esa posición, trata de resistir los intentos de quien lo examina para desplazar el pulgar hacia la palma de la mano v luego a supinarlo nuevamente hacia la mesa. Si el abductor esta débil será difícil para el paciente la abducción del pulgar. Si el oponente está débil ello se demuestra por resistencia disminuida a la rotación hacia la mesa del pulgar en abducción.

La debilidad motora demostrada por cualquiera de estas dos pruebas indica compromiso neural avanzado.

\section{Pruebas electrofisiológicas}

Las pruebas electrofisiológicas constituyen el único medio objetivo para evaluar la función nerviosa. Las velocidades y latencias de conducción del nervio 
mediano pueden compararse con aquellas publicadas para la población general, con las del mediano contralateral o con las de otros nervios de la misma extremidad, o con valores obtenidos previamente en el mismo paciente. Estas comparaciones pueden estar complicadas por enfermedades sistémicas o por alteraciones en la conducción nerviosa dependientes de la edad. Este detecta la disminución de la velocidad de conducción sensitiva y motora. Es útil para confirmar el diagnóstico y valorar la severidad de la compresión: si es normal, no descarta STC. Una latencia motora distal de más de 4.5 milisegundos o una latencia sensorial de más de 3.5 milisegundos es considerada anormal. También, cuando el tiempo de conducción en el nervio mediano en una extremidad es comparada con el de la extremidad contralateral o con el del nervio cubital del mismo lado se diferencian por más de 1.0 milisegundo para la conducción motora, o de más de 0.5 milisegundos para la conducción sensorial se consideran anormales.

\section{Variaciones del Cuadro Clínico}

\section{Leve o Agudo (Etapa I)}

En estos casos se produce adormecimiento y dolor en la zona inervada por el nervio mediano. Se ve disminuida la sensibilidad de los dedos pulgar, índice y medio de la mano afectada y en ocasiones también la parte radial del dedo anular. Los síntomas se presentan por lo general en la noche y se ha documentado que el dolor, la sensación de adormecimiento y hasta los calambres, despiertan al paciente más de una vez durante la noche.

El STC agudo ocurre rara vez, generalmente se da a consecuencia de un trauma, o después de realizar una actividad de gran esfuerzo con la utilización de miembros superiores y movimientos repetitivos, con resistencia de flexo extensión de muñecas, que la persona por lo regular no está acostumbrada a llevar a cabo, y se caracteriza por un desarrollo rápido de síntomas marcados. Entre sus causas se hallan las fracturas o dislocaciones de muñeca, estados hemo- rrágicos (usualmente en pacientes anti coagulados) o la inmovilización con yesos en posiciones extremas de fracturas de la muñeca, que se constituyen en un síndrome compartimental agudo, generando a menudo una urgencia quirúrgica.

\section{Moderado (Etapa 2)}

Se presenta un marcado compromiso de la sensibilidad y movilidad de la mano afectada.

Esta etapa es más común que la anterior, y puede clasificarse en Temprano, Intermedio o Tardío. Los pacientes con STC moderado temprano, sufren de dolor intermitente y parestesias que empeoran todas las noches o como consecuencias de actividades específicas. Las pruebas electromiográficas se encuentran mostrando alteraciones y las latencias sensoriales se prolongan más a menudo que las motoras sin que los pacientes presenten todavía anomalías del músculo tenar. Aun cuando el nervio mediano no muestra cambios morfológicos evidentes en esta fase, sí sufre de una isquemia epineural reversible lo mismo que de un trastorno axonal.

Los pacientes con STC moderado intermedio experimentan dolor, parestesias y adormecimientos de los dedos más constantes. Los valores para las pruebas de umbral sensorial y de latencias motrices dístales están aumentados. Se observa un compromiso persistente de la circulación intraneural, lo mismo que el edema epineural e intrafascicular, eventos que hacen poco factible la obtención de una respuesta beneficiosa duradera utilizando un tratamiento conservador; se indica entonces, la liberación el túnel carpiano.

\section{Severo (Etapa 3)}

Se presenta en esta etapa un bloqueo de la conducción sensitiva y atrofia de la parte motora. Se debe realizar cirugía con liberación del nervio mediano a través de la ruptura del ligamento transverso del carpo. Los pacientes con STC severo presentan pérdida permanente de las funciones sensorial y motora de la parte del mano inervada por el nervio mediano además de atrofia de los músculos de la eminencia tenar. Las pruebas de umbral, de densidad 


\section{ARTICULOS INÉDITOS}

y las de innervación son anormales. Las velocidades de conducción nerviosa están disminuidas y las de latencias distal sensorial y motriz aparecen prolongadas. El edema endoneural de larga duración, eventualmente llega a producir fibrosis del nervio mediano así como la desmielinizacion parcial y degeneración axonal de dicho nervio. La mayoría de estos pacientes llegan a tener resolución completa o parcial de sus síntomas con la liberación del túnel carpiano, aunque en algunos el daño ya es irreversible.

\section{Tratamiento no Quirúrgico o Conservador}

Éste se puede considerar teniendo en cuenta la causa subyacente que esté produciendo el STC. Si existe una enfermedad sistémica de base como la diabetes, la artritis reumatoidea, el hipotiroidismo o cualquier otra enfermedad asociada, su tratamiento médico deberá ser prioritario. El tratamiento conservador esta indicado para aquellos pacientes quienes presentan molestias o síntomas sensoriales subjetivos. Aquellos que ya presentan cambios neurológicos objetivos, como debilidad, atrofia de la eminencia tenar y rigidez de los dedos tienen una lesión más destructiva y requieren de una descompresión quirúrgica. El tratamiento conservador se inicia usualmente en el consultorio del médico con inyecciones locales de esteroides, medicación oral anti-inflamatoria, se ha utilizado también la formulación oral de vitamina B6 (Vitamin B6 therapy for carpal tunnel syndrome. Jacobson, M.D. Hand Clinics, Mayo de 1996), y la instrucción al paciente del reposo de la mano, para ello es remitido a Terapia Ocupacional. Una vez en el servicio de Terapia Ocupacional, además de diseñar, elaborar y aplicar ferulaje que inmovilice la muñeca del paciente, le da indicaciones de técnicas de protección articular, higiene postural, relajación y ahorro de energía.

\section{Tratamiento Quirúrgico}

La cirugía es una opción cuando el síndrome no responde bien al tratamiento conservador. Debe seccionarse quirúrgicamente todo el ancho del ligamento transverso del carpo. Es decir, a través de la cirugía el médico cirujano de mano realiza una sección del ligamento carpiano trasverso, aumentándose el tamaño del túnel y dando como resultado la reducción de la presión intersticial.

\section{INTERVENCIÓN dE TERAPIA OCUPACIONAL}

El Terapeuta Ocupacional debe realizar el proceso de valoración ocupacional completo con una anamnesis del paciente a través de la entrevista personal en donde se realiza una historia ocupacional. En esta última, se indaga acerca de la ocupación que realiza el paciente, los equipos que utiliza, así como las herramientas que son propias de su oficio. Se determina, si este es un trabajo pesado, moderado o liviano; la jornada laboral, los descansos que se toman durante la misma y la posición que debe mantenerse para la elaboración de dicha ocupación. Además, se recrean las actividades que se llevan a cabo en el trabajo del paciente. Se pregunta el tiempo que lleva desarrollando la actividad actual, y se hacen cuestionamientos acerca de la actividad realizada inmediatamente anterior a la actual, tratando de encontrar alguna relación entre los síntomas del momento y la ocupación (Protocolo de intervención para síndromes de sobreuso. Hospital Militar Central).

Se determina también la funcionalidad e independencia en el desempeño de las actividades de la vida diaria, haciéndole énfasis especial al paciente para que mencione aquellas actividades de autocuidado o de procedimiento donde tiene dificultad o siente dolor en las manos o antebrazos.

El signo con mayor valor predictivo, pero el menos utilizado es el de Flick. (Rodríguez. Síndrome del Túnel Carpiano. Guías Clínicas 2001.) Éste es positivo cuando el paciente al ser preguntado: "¿Qué hace usted con la mano cuando los síntomas están peor?" responde agitando su mano de la misma manera que lo hace para bajar un termómetro.

También, se determina mediante pruebas más 
objetivas como la goniometría, la amplitud articular activa y pasiva de muñecas y dedos observándose si el edema ha ejercido su influencia sobre los arcos de movimiento.

Se realizan pruebas que determinan la capacidad del paciente para realizar los patrones integrales, en especial los agarres medianos y livianos, ya que éstos se llevan a cabo gracias a la acción de la flexión de los cuatro últimos dedos y la flexión y oposición del pulgar, acciones que son llevadas a cabo por los músculos inervados por el nervio mediano.

Una vez determinada la capacidad para la realización de los diferentes patrones integrales, se determina con qué fuerza se realizan los agarres y las pinzas, utilizando el dinamómetro y el pinzometro. Si no se tiene la posibilidad de acceder a estos equipos, se realizará un examen muscular manual de los músculos de la eminencia tenar y los flexores de los dedos. Se deben realizar también las pruebas de sensibilidad, llevándose a cabo la prueba de Weber o de discriminación de dos puntos, y la de monofilamentos. Además se aplican las pruebas de provocación es decir las pruebas de Tinnel y de Phalen, todas éstas explicadas atrás.

Por último, se debe llevar a cabo la valoración de las molestias subjetivas que tiene cada uno de los pacientes, a través ya sea de la escala análoga del dolor o mejor, usando diferentes formatos de valoración de los síntomas como adormecimiento en el día, medidos por cantidades de veces, en la noche por el número de veces que se despierta por esta causa, posición de las manos al dormir, y así con el dolor, los calambres, el cosquilleo y otras molestias.

El tratamiento ofrecido por el Terapeuta Ocupacional a los pacientes con STC se realiza según la etapa en que se encuentre el usuario en el momento de la remisión al servicio, y se basa en el enfoque Biomecánico, principalmente. A continuación se mencionan las etapas.

\section{Etapa 1 Aguda}

El objetivo es minimizar los efectos de la inflamación, reducir el dolor y mantener el deslizamiento normal de los tendones flexores dentro del túnel carpiano mediante un programa de ferulaje, modalidades físicas y ejercicio terapéutico.

$\mathrm{Si}$ existe una actividad específica que cause o agrave el Síndrome del Túnel del Carpo, el Terapeuta Ocupacional recomendará modificar la forma de realizar estas actividades siguiendo las indicaciones listadas a continuación.

- Alternar las tareas, mejor que realizar una sola durante un largo periodo de tiempo.

- Hacer descansos y posteriormente ejercicios de estiramiento cada 20 o 30 minutos cuando se realice un trabajo altamente repetitivo.

- Si el STC tiene relación con el trabajo, intentar modificar las condiciones de éste para disminuir la irritación del nervio.

\section{UTILIZACIÓN dE FÉRULAS dE MUÑECA}

La férulas que inmovilizan la muñeca son especialmente efectivas para disminuir las sensaciones nocturnas de adormecimiento y hormigueo en la mano. Cuando la muñeca se flexiona o extiende al máximo por mucho tiempo, el túnel del carpo se hace más estrecho; manteniéndose una posición neutra de muñeca, el túnel del carpo alcanza su máximo diámetro. Por tanto, mantener la muñeca en posición neutra, evitando que se flexione o extienda por la noche puede reducir los síntomas.

la férula se usa para inmovilizar en posición neutra la muñeca y así reducir la presión sobre el nervio mediano, ampliando el espacio del túnel. Se puede elaborar palmar o dorsal, pero en todo caso el puño debe inmovilizarse en dicha posición. El horario de uso depende de la severidad de los síntomas. La férula es útil para aliviar el dolor nocturno y disminuir las parestesias que resultan de dormir con las muñecas en flexión extrema. El nervio mediano es sometido tres veces más a presión cuando se hace flexión de puño prolongadamente que cuando el puño está en neutro o extendido ligeramente. Se prescribe uso continuo diurno y nocturno de la férula de 4 a 6 semanas, excepto para higiene y la realización de los ejercicios. El tiempo de uso se va disminuyendo lentamente, 
tan pronto la inflamación y los síntomas empiezan a disminuir.

Dentro de las modalidades terapéuticas utilizadas para el problema inflamatorio están: el uso de paquetes congelados, los baños de contraste o la introducción alternada de las manos en líquidos fríos y calientes, circunstancias que se pueden aprovechar en actividades con propósito especialmente diseñadas y graduadas por los Terapeutas Ocupacionales. La estimulación eléctrica y la fonoforesis, aunque son técnicas utilizadas por Terapia Física, en países como Estados Unidos son usados también por Terapia Ocupacional (Acute carpal tunel Síndrome. Revista Hand Clinics, Agosto de1998.). Sin embargo, en nuestro medio son técnicas aplicadas por estos profesionales exclusivamente, a menos que se tenga entrenamiento en su utilización o una especialización en miembros superiores.

Una vez la inflamación disminuye la excursión restringida de los tendones puede ser tratada con calor, dentro de actividades con propósito que impliquen su uso, seguido por ejercicios de estiramiento de flexores extrínsecos y deslizamiento aislado de los tendones flexores de los dedos, incrementándose así la extensibilidad de los flexores y promoviéndose la excursión normal de estos tendones. Además, se recomienda aprender las técnicas de protección articular y de conservación de energía .

\section{TÉCnicas de conservación de ENERgía}

Son guías para la ejecución de importantes tareas de la vida cotidiana, de tal forma que se conserva la energía personal, se simplifica el trabajo, se maneja el tiempo adecuadamente y/o se organiza el ambiente para minimizar el gasto de energía y que pueden ser utilizadas en todas las actividades básicas cotidianas

\section{Principios- técnicas generales:}

- Planear con anterioridad.

- Organizar el trabajo antes de la iniciación.

- Delegar actividades a otras personas

- Eliminar tareas innecesarias.

- Sentarse en lo posible a trabajar.
- Combinar tareas para eliminar trabajo extra.

- Descansar antes de la fatiga.

- Si es necesario, pedir ayuda a un tercero

- Dormir adecuadamente

\section{Técnicas específicas:}

- Usar elementos eléctricos para conservar la energía personal

- Usar utensilios y herramientas livianas.

- Trabajar con la gravedad asistida y no resistida.

\section{Principios de la protección articular}

El propósito final es reducir el estrés extremo aplicado en las articulaciones que presentan alguna alteración durante las actividades de la vida diaria. Si hay dolor durante la realización de una actividad significa que la articulación está empeorando y puede incrementarse la inflamación. Realizar la actividad sin dolor significa que se esté ayudando a la articulación. Las técnicas de protección articular son métodos modificados para ejecutar las actividades reduciendo el estrés y la fuerza sobre las articulaciones. Se deben tener en cuenta algunos principios que ayudarán a reducir el dolor y a sentirse mejor al realizar las actividades cotidianas. El concepto de protección tendinosa presentado por Walker y Rhem, a principios de los noventa, consiste en instruir al paciente en evitar las posturas del puño que incrementa la presión sobre el nervio mediano.

Luego se encontro que la flexión de puño, la desviación cubital y la flexo-extensión repetitiva de la muñeca con agarres digitales y de pinzamientos, son los movimientos más agravantes.

Las actividades que requieren estos movimientos son: martillar, abrir válvulas o tapas de frascos, ajustar con alicates, serruchar y girar manijas. Una vez las actividades son identificadas, se instruye al paciente para modificar la forma en que se realiza.

- Evitar posiciones excesivas o constantes que presionen las articulaciones.

- Evitar posiciones estáticas prolongadas.

- Usar adaptaciones en utensilios para la realización de tareas.. 
- Respetar el dolor y reconocer la fatiga.

- Distinguir entre el malestar habitual generado por la enfermedad y la fatiga generada por una actividad.

- Eliminar actividades si su ejecución generan dolor persistente.

\section{ETAPA 2: FortalecimiEnTO - RETORNo AL trabajo}

Empieza de forma gradual la introducción de ejercicios resistidos para incrementar la fuerza. Todos los ejercicios de fortalecimiento están precedidos por un programa de calentamiento de movimiento activo del pulgar, el puño y los dedos al igual que tres minutos de trabajo aeróbico, Inicialmente se utilizan los ejercicios isométricos para incrementar la fuerza sin incrementar la inflamación e incrementar los síntomas sensitivos. Los ejercicios isométricos son llevados a cabo dos veces por día por veinte repeticiones.

Cuando el paciente demuestra un edema mínimo y excursión total de los tendones, mientras completa 20 repeticiones de ejercicios isométricos, los ejercicios de repetición isotónicas son iniciados.

Los ejercicios isotónicos se inician para la flexoextensión de puño y desviación radial y cubital. La técnica modificada de Oxford de ejercicios resistidos regresivos se recomiendan para permitir al paciente incrementar la resistencia sin experimentar el nivel de fatiga reportado después de la ejecución de ejercicio resistido progresivo. La resistencia es ganada cuando el paciente realiza la simulación del trabajo.

Está documentada la utilización de baterías o equipos para simular trabajos y para realizar ejercicios isotónicos o isométricos según la necesidad; sin embargo, en nuestro medio no se usan con frecuencia. El equipo terapéutico de simulación del trabajo (BET), es una batería que utiliza herramientas que simula una actividad laboral.

El paciente debe ser educado para modificar métodos de ejecución que pueden disminuir el estrés ergonómico en el ambiente laboral y así minimizar la recurrencia del STC.

\section{FÉRULAS}

La férula es un aparato ortopédico que es aplicado en el miembro superior de una persona para brindar soporte de alguna estructura, para lograr el posicionamiento de esta, para inmovilizar, para corregir deformidades, para asistir a un músculo que se encuentra débil o para restaurar alguna función, y hace parte de un programa de tratamiento.

La férula tradicional para el Túnel del Carpo y más comúnmente utilizada es una férula corta, volar, elaborada en material termoplástico moldeable a bajas temperaturas, que mantiene la articulación de la muñeca en $10^{\circ}$ a $20^{\circ}$ de extensión, pues según estudios se ha comprobado que en esta posición, y con uso permanente los pacientes tienen disminución en la sintomatología, permitiendo la oposición del pulgar y la movilización libre de todos los demás dedos. La férula tradicional, palmeta corta para el STC, estabiliza la muñeca, manteniéndola en la posición ya mencionada. Su uso reduce el dolor y la inflamación dentro del túnel.

Las condiciones de utilización de esta férula son determinadas por la etapa en que se encuentre la patología. Respeta el pliegue distal de la mano, se localiza por debajo de la cabeza de los metacarpianos, Es corta, y contornea el antebrazo hasta la unión del tercio medio con el tercio proximal del mismo. Sus medios de sujeción son dos abrazaderas elaboradas en velcro de $2,5 \mathrm{cms}$. de ancho, uno a nivel proximal y otro a nivel distal por debajo de la articulación de la muñeca. Y una barra metacarpiana, hecha también en material termoplástico, que sale por el primer espacio interdigital y se dobla hacia el dorso de la mano, aproximadamente hasta el borde cubital del segundo metacarpiano, terminando en un velcro que va hasta el tope cubital de la férula, un poco por debajo de la articulación metacarpofalángica del dedo meñique.

La persona deberá poder hacer oposición del pulgar en octavo grado (hasta la falange proximal del meñique), conservándose así la funcionalidad de la mano. 
La férula en material termoplástico se elabora en promedio para cada mano en noventa minutos, es decir tres sesiones de terapia convencional. El material, incluyendo el velcro y los remaches que se usan para fijar los velcros, es en la actualidad de $\$ 45.400$.oo pesos moneda corriente. Este valor sumado al de las tres sesiones, arroja un costo total aproximado de $\$ 63.250 .00$. Éste valor debe ser cancelado o por el paciente o por su Empresa Prestadora de Salud.

La férula "Manu" para pacientes con STC es hecha de material suave, sin componentes rígidos sujetada con correas de velcro alrededor de las cabezas de los cuatro últimos metacarpianos. Está constituida por un rectángulo del tamaño de los cuatro últimos dedos, donde por una hendidura horizontal, del tamaño del ancho de los dedos tercero y cuarto, se puedan introducir los mismos, quedando por encima, y dejando los dedos segundo y quinto, por fuera y por debajo. Por último, se adhiere otra correa de velcro a nivel de la muñeca que evitará que se corra la férula. Se coloca un componente en forma rectangular del tamaño del ancho del antebrazo, que conecte la correa de la muñeca y el rectángulo de los dedos.

La férula deja libre al pulgar y permite la flexo extensión de la muñeca, sin embargo produce presión suave sobre la cabeza de los metacarpianos del quinto y segundo dedos, y hala discretamente los dedos tercero y cuarto, disminuyendo síntomas como las parestesias y dolores a nivel de toda la mano.( Ann Innovative Hand Brace For Carpal Tunnel Syndrome: A Randomized Controlled Trial. Manente, M.D., Muscle And Nerve Journal, Agosto de 2001).

La Férula se elabora en papel plástico espumoso de cuatro milímetros de espesor, conocido comercialmente como Fommy o Fanty

El material, incluyendo el velcro y el pegante instantáneo que se usan para fijar los velcros, tiene en la actualidad un valor de $\$ 3.700$.oo pesos moneda corriente. Este valor sumado al de las dos sesiones que se tarda un Terapeuta en su elaboración, arroja un costo total aproximado de $\$ 15.600$.oo Este valor debe ser cancelado o por el paciente o por su Empresa Prestadora de Salud.

\section{Conclusiones}

- El STC es el trastorno de trauma acumulativo mas frecuente.

- La causa más frecuente del STC es la tenosinovitis estenosante de los tendones flexores de los dedos, por lo general bilateral, que impide su deslizamiento a través del túnel. Las malas posturas y la no adecuada utilización de las diferentes partes de las computadoras, elemento esencial de trabajo, han incrementado el número de pacientes con STC.

- En la población general alrededor del 15\% de las personas manifiestan sintomatología compatible con STC. Se presenta en una proporción de mujeres a hombre de 8:1. La ocupación laboral tiene estrecha relación con la aparición del STC, en especial, la ejecución de acciones manuales repetitivas, en nuestro medio específicamente ocupaciones como las de procesamiento de comidas, manufacturas, trabajo de construcción, costura y trabajo en computador o deportes. Las mujeres con mayor incidencia son aquellas entre los 40 y 60 años de edad.

- El STC está influenciado por la nueva tecnología y por el aumento de trabajo físico, movimiento y esfuerzo concentrado en la muñeca además del mal diseño de los equipos y herramientas.

- El STC tiene una causa psicológica, tanto que es catalogada como una enfermedad de tipo social. Los psicólogos aseguran que a pesar de que el STC es una afección física, los factores psicológicos contribuyen a su desarrollo y mantenimiento.

- Los síntomas varían de agudos a crónicos, pero en general se acentúan en las noches, produciendo disestesias, calambres y adormecimiento de la zona inervada por el mediano.

- Para el diagnóstico se realizan pruebas de provocación, de sensibilidad y motrices. Se hacen por lo general electromigrafias para verificar la velocidad de conducción nerviosa del mediano.

- Terapia Ocupacional intervine a nivel preventivo, en etapa aguda, intermedia o crónica. 
- Para la valoración se realiza historia ocupacional, pruebas de provocación, pruebas de sensibilidad y motrices. Se hace énfasis en la funcionalidad e independencia del usuario en la realización de las actividades de la vida diaria y las consecuencias en la causación personal.

- Para la intervención se aplican técnicas del enfoque Biomecánico principalmente, donde se instruye al paciente en técnicas de ahorro de energía, de protección articular, fortalecimiento y reentrenamiento adecuado y se le aplica ferulaje tradicionalmente de palmeta corta en material termoplástico, de uso permanente por tres semanas con resultados adecuados, teniendo en cuenta la disminución o abolición de la sintomatología.

- La férula tradicional tiene un costo mayor que la Manu. Aunque los resultados no están escritos en Colombia, los pacientes que las han usado refieren disminución o abolición de la sintomatología.

- Se está implementando el uso de la Férula "Manu" en papel Foamy, a un costo aproximadamente cinco veces menor que la tradicional, sin resultados escritos en Colombia, pero los pacientes que las han usado han referido disminución o abolición de la sintomatología.

- Se deben realizar más estudios que demuestren cuantitativamente la eficiencia y eficacia de la utilización de los dos tipos descritos de ferulaje y el beneficio del costo.

\section{Referencias Bibliográficas}

Ann Innovative Hand Brace For Carpal Túnel Síndrome: A Randomized Contolled Trial. Manente, M.D. , Muscle And Nerve Journal, Agosto De 2001 Pags. 1020 - 1025

Acute Carpal Tunnel Syndrome. Szabo, M.D. Hand Clinics. Vol. $14 N^{\circ} 3$ Agosto De 1998. Pags 419. 427

The Role Of Splinting And Rehabilitation In The Treatment Of Carpal And Cubital Túnel Síndromes. Sailer, O.T. Hand Clinics, Vol.12, N², Mayo De 1996. Pags 223 - 240.

The Pathophysiology Of Carpal Tunnel Syndrome. Kerwin, M.D. Hand Clinics, Vol. $12 \mathrm{~N}^{\circ} 2$ Mayo De 1996. Pags. 243 -249 .

Vitamin B6 Therapy For Carpal Tunnel Syndrome. Jacobson, M.D. Hand Clinics, Vol $12 \mathrm{~N}^{\circ}$ 2, Mayo De 1996. Pags. 253 $-257$.

Carpal Túnel Síndrome . Von Schroeder, M.D. Hand Clinics, Vol 12, N 4 Noviembre De 1996.
Comparacion De Dos Ferulas De Distinto Material En Cuanto A Efectividad En Amas De Casa Con Stc. Santaella Y Herrera, T.O. Revista De La Ocupación Humana, Vol. $7 \mathrm{~N}^{\circ} 4$ De 1998. Pags $13-37$

Actualización En Cirugía De Mano, Iladiba,. M.D. Volumen 16, Num. 9,- 2002 Pags. 44-46.

Overuse Syndrome/ Repetitive Strain Injury: The Occupational Therapit'S Role. Mcnaugthon, M. D., British Journal Of Occupational Therapy. Occupational Febrero De 1997.

Guia De Manejo Para El Síndrome De Tunel Del Carpiano, Szabo Robert, Tribuna Medica. Vol.91 Mayo De1998. Pag. 265-268.

Rodríguez. Síndrome Del Túnel Carpiano Guías Clínicas 2001; 9 De Marzo De 2000.

Hand The: Examination And Diagnosis American Society For Surgery Of The Hand. 1997.

Cirugía De La Mano Bunnell-Boyes.. Editorial Intermedica, 1967.

Ndromes Dolorosos De La Mano. Cailliet, Rene. Síeditorial El Manual Moderno.

Fisioterapia De La Mano. Christine, A. Moran. Editorial Jims. Barcelona, España.

Assistive Technologie Principles And Practice.Cook, Hussey, Hand Rehabilitation In Occupational Therapy Cromwen, Florence.

Hand Splinting Principles And Methods Fees, Philip.

Hand Surgery. Flynn, J. Edward. Editorial El Ateneo. Buenos Aires

Rehabilitation Of The Hand: Surgery And Therapy Hunter, Mackin, Callahan.. Fourth Edition.

Occupational Hand Upper Extremity Injuries, Diseases. Kasdan. Morton,L. Mosby 1991.

Kinesiología Y Anatomía Aplicada Rasch \& Burke, 1996

Concepts In Hand Rehabilitatión. Stanley, Barbara. Davis 1992

Lesiones Agudas De La Mano.Wolfort, Francis. 